\title{
Michel Offerlé (dir.), Patrons en France
}

\section{Pierre-Marie Chauvin}

\section{OpenEdition}

\section{Journals}

Édition électronique

URL : http://journals.openedition.org/travailemploi/7738

DOI : 10.4000/travailemploi.7738

ISSN : $1775-416 \mathrm{X}$

\section{Éditeur}

DARES - Ministère du Travail

\section{Édition imprimée}

Date de publication : 1 juillet 2017

Pagination : Paris, La Découverte, 2017

ISSN : 0224-4365

\section{Référence électronique}

Pierre-Marie Chauvin, « Michel Offerlé (dir.), Patrons en France », Travail et Emploi [En ligne], 151 | juilletseptembre 2017, mis en ligne le 11 juillet 2019, consulté le 25 septembre 2020. URL : http:// journals.openedition.org/travailemploi/7738; DOI : https://doi.org/10.4000/travailemploi.7738 


\section{Patrons en France}

\section{Michel Offerlé (dir.)}

Paris, La Découverte, 2017, 660 p.

\section{Lu par Pierre-Marie Chauvin*}

Patrons en France. Le titre donne l'impression d'une objectivité froide, à la fois révélatrice et trompeuse quant au contenu du livre. Révélatrice car il s'agit d'une livraison presque brute d'interviews patronales, donnant à voir l'intérieur d'un monde plus souvent désigné, apostrophé ou caricaturé qu'étudié. Trompeuse, car cette idée de monde est à entendre dans un sens principalement subjectif et narratif, et n'a donc rien de froid : les discours patronaux prennent la forme de mises en récit de soi et de son entreprise, grâce auxquels ces patrons sont montrés sous un jour évidemment « humain ». Ils parlent de leurs motivations, de leurs difficultés, de leurs ambivalences, et plus généralement du sens qu'ils donnent à leur activité. Le lecteur militant, qu'il soit bouffeur de patrons ou suppôt du capital, y trouvera peut-être du grain à moudre, mais ce sera alors grâce aux filtres d'une lecture très sélective et partiale, car rien n'est monolithique dans ce livre. Les patrons forment-ils un groupe social ? Il apparaît, du point de vue des acteurs patronaux eux-mêmes, qu'ils n'en forment pas un, et encore moins une classe ou un camp. Pierre Bourdieu qualifierait probablement ce discours de dénégation catégorielle au service du pouvoir, mais malgré un usage répété du mot «capitaux » (plutôt que capital) dans le livre, les discours patronaux n'y sont pas réduits à une position de classe. Dès lors que l'on prête attention à la diversité des trajectoires et à leurs polyphonies, difficile de voir dans la mosaïque de témoignages un groupe bien homogène d'ennemis de classe !

Le livre présente une collection d'entretiens réalisés par un collectif de chercheurs dont la principale tâche d'écriture a été de proposer une contextualisation empirique et théorique des entretiens cités. Michel Offerlé, qui a coordonné l'ouvrage et animé le séminaire à sa source, prolonge ainsi son œuvre, rare en France, consacrée au patronat comme acteur social. Après avoir étudié le patronat comme acteur collectif ${ }^{1}, \mathrm{M}$. Offerlé et les 28 auteurs de l'ouvrage (étudiants, doctorants et chercheurs confirmés) poursuivent cette dynamique de recherche à une échelle plus fine, celle des individus. Au moment où les sciences sociales s'interrogent sur le partage des données et l'open

\footnotetext{
* Sorbonne Université.

1. Voir notamment le récent Les Patrons des patrons, histoire du MEDEF, 2013, Odile Jacob.
} 
data, ils transforment ce qui aurait pu simplement être un matériau mis en ligne en acte éditorial fort, mais sans thèse.

Acte éditorial fort car le livre n'est pas uniquement un aquarium géant (657 pages, tout de même) présentant un matériau brut de subjectivités patronales. Il offre également une introduction générale, un court chapitre donnant quelques éléments statistiques sur les mondes patronaux (fondé sur les Enquêtes Emploi de l'Insee), des introductions de parties présentant brièvement les entretiens à suivre (six parties ${ }^{2}$ ) et de nouvelles introductions précédant chaque entretien. Enfin, une conclusion à la densité bienvenue (40 pages) vient clore l'ensemble. Cela suffit-il, cependant, à faire de cet ouvrage autre chose que la restitution d'une démarche entomologique ? Quel est donc l'apport de cet ensemble original ?

Le livre vaut moins pour une thèse que par son geste. Geste de dévoilement d'une parole patronale personnelle, peu audible dans le paysage médiatique et scientifique, en tout cas sous cette forme, longue et nuancée. Il prolonge ainsi la littérature sociologique consacrée au patronat et aux entrepreneurs, en offrant un matériau empirique inédit par son volume et sa variété. Ce faisant, le livre entend prendre le contre-pied de regards courants sur les acteurs patronaux en les montrant hétérogènes, non obsédés par le profit (ou en tout cas présentant également d'autres logiques d'action) et appuyés sur des collectifs, plus que self made men isolés du monde. Ainsi le livre permet-il de dégager une diversité des profils et des motivations là où l'on ne voit couramment que de l'homogène, des appuis collectifs là où l'on ne voit que de la réalisation de soi, des récurrences là où l'on ne voit que des singularités. Le plus difficile est probablement de repérer ces récurrences, car le travail est à faire par le lecteur. Il doit ainsi s'en remettre à sa propre mémoire pour faire des liens, identifier des résonances, établir des parallèles, et éventuellement dessiner des idéaux types. À défaut d'esquisser ces types, nous pouvons relever trois exemples, parmi une multitude d'éléments présents dans l'ouvrage, de ce qui rassemble et de ce qui peut opposer les patrons interviewés, en termes de valeurs, de temporalités et de motifs.

Les premiers points communs qui apparaissent nettement sont des répulsions partagées : rejet des acteurs politiques ( « déconnectés des réalités de l'entreprise »), notamment de gauche (mais pas exclusivement), rejet de certains dispositifs comme « les 35 heures », rejet des horaires fixes, rejet des « glandeurs » et des « fonctionnaires », etc. De manière plus positive, tous les acteurs interrogés soulignent l'importance de la « valeur travail » et plus précisément du « travail sans compter ». Ils reconnaissent la porosité des frontières entre temps professionnel et temps privé et valorisent une forme de disponibilité permanente, tout en regrettant les effets psychologiques qu'elle peut générer : les « angoisses »- le terme revient à plusieurs reprises - du chef d'entreprise.

2. Les entretiens sont regroupés en six grandes parties thématiques dont le découpage est évoqué plus que justifié en introduction : « Trajectoires entrepreneuriales »; « Surfaces patronales »; « Le puzzle du capital entrepreneurial »; « Diriger une entreprise »; « Valeurs de l'argent »; « Goûts, valeurs et opinions ». 
Parmi les pistes les plus stimulantes de l'ouvrage figure précisément la question des temporalités patronales. La conclusion revient ainsi sur le fait que la maîtrise de son temps, tout en étant une préoccupation commune aux acteurs interrogés, n'est pas distribuée également dans l'espace patronal. L'engagement dans le temps long, les facultés d'anticipation, l'aisance dans les bifurcations ou encore la longévité entrepreneuriale sont des enjeux temporels face auxquels les acteurs interrogés ne sont pas égaux, en fonction de leurs appuis sociaux et de leurs dynamiques. Selon la santé économique de leur entreprise, selon leurs ressources financières, familiales et relationnelles, les facultés de projection, de bifurcation stratégique, ou de pérennisation de leur situation patronale sont hétérogènes. Certains naviguent dans le passé comme dans l'avenir de leur entreprise de manière aisée, d'autres soulignent la difficulté de certaines transitions, d'autres encore annoncent la fin programmée de leur activité.

En observant les différentes motivations affichées dans les entretiens, on comprend que « devenir patron » est rarement une finalité en soi, si ce n'est sous la forme d'une revendication d'autonomie ( « devenir son propre patron ») - récurrence bien identifiée par la littérature sur l'entrepreneuriat et ici confirmée par la plupart des entretiens. Ce qui réunit ces patrons, c'est le fait de s'affranchir du lien de subordination salariale, mais avec des marges de manœuvre inégales dans leurs relations avec d'éventuels actionnaires, banquiers, salariés, clients, partenaires, fournisseurs, etc. Certains entretiens montrent des acteurs fixant les grandes orientations de leur entreprise, libérés du « sale boulot », de la gestion courante et des détails du quotidien, tandis que d'autres sont des « acteurs-à-tout-faire », véritables couteaux suisses de leur entreprise, notamment lorsqu'ils ont gravi les échelons internes avant d'endosser des fonctions de direction. Si l'on en croit la plupart des discours cités, le profit comme source de motivation, et plus généralement l'argent comme résultat anticipé de l'action entrepreneuriale, ne seraient que des produits secondaires, advenant de surcroit, et non des moteurs initiaux. Cependant, le rapport à l'argent n'est pas uniforme, étant plus ou moins présent dans les discours et sous différentes modalités : préoccupation permanente, fierté d'un chiffre d'affaires ou d'un niveau de rémunération, souci d'un réemploi productif et utile de l'argent accumulé, etc.

Au final, que penser de ce projet éditorial ? S'il est inédit par son objet, il prolonge une tradition de matériaux biographiques commentés sous la forme de livres, du Paysan Polonais de William Thomas et Florian Znaniecki à La Culture des individus de Bernard Lahire, en passant par La Misère du Monde de Pierre Bourdieu (pour aller très vite). Ce type d'ouvrage vaut autant pour son contenu vivant que pour les paragraphes analytiques qu'il contient généralement. Dans Patrons en France, ces commentaires sont essentiellement des introductions - de partie et d'entretiens - dont le statut est hybride : présentation de la personne interviewée ${ }^{3}$, esquisse d'analyse ${ }^{4}$ et

3. Caractéristiques sociodémographiques, histoire entrepreneuriale, capitaux économiques et sociaux, etc.

4. Étude de l'ethos entrepreneurial, place du profit dans la logique d'action, identification des appuis à la création

d'entreprise, analyse du jeu avec les espaces sociaux et géographiques, etc. 
moment de réflexivité méthodologique 5 . Ils sont à la fois importants par les éclairages qu'ils offrent et limités par leur aspect non systématique et fragmentaire. L'information biographique disponible à propos des interviewés varie beaucoup en fonction des interviews, et la façon dont elle est relatée également. Certains termes théoriques sont répétés (« ethos », « capitaux », « espaces », « trajectoires », « appuis », « bifurcations », «indépendance », etc.) sans être définis ni reliés par une théorie générale ou, à tout le moins, par un cadre théorique minimal. Un flottement problématique concerne par exemple l'usage des termes de "patrons » et d' «entrepreneurs », évidemment centraux, et utilisés de manière substituable dans l'ouvrage. Pourtant, certains interviewés s'emploient à établir eux-mêmes des distinctions, par exemple lorsque le patron de Saint-Gobain assure n'être ni un créateur d'entreprises, ni un gestionnaire, dans la mesure où son activité consiste à dessiner les grands caps d'une entreprise porteuse d'une histoire et d'une tradition qu'il lui faut adapter aux enjeux contemporains. Son activité repose notamment sur l'amplitude du paquebot considéré, représentant « 200000 personnes » et « 40 milliards ». Un effort de distinction appuyé sur ce type d'auto-catégorisation aurait été utile en début d'ouvrage, éventuellement croisé avec les distinctions existantes dans la littérature sociologique. Un autre aspect récurrent mais non creusé est la notion d' « ethos entrepreneurial » (p. 18) : elle se trouve incarnée par une variété de formes empiriques qu'il aurait été intéressant de rassembler et confronter. Par exemple, l' « ethos de la direction, où on doit taper du poing sur la table » (p. 61) n'a probablement pas grand-chose à voir avec l'ethos du créateur, mû par un esprit d'initiative et d'innovation (que l'on retrouve à plusieurs moments dans l'ouvrage), ou avec l'ethos clivé d'un entrepreneur migrant composant avec des liens communautaires, ou encore avec celui d'une entrepreneure libraire sans revenus ou presque, assimilable aux « entrepreneurs passionnés » étudiés par Fanny Dubois et Philippe Terral ${ }^{6}$.

Enfin, d'un point de vue méthodologique, quelques questions subsistent sur les entretiens réalisés, alors même que les auteurs assument en introduction le «pari d'exposer à la critique notre matériau (l'entretien - ou plutôt un extrait ou des extraits - avec ses questions et ses réponses) » (p. 17). Comment ont été réalisés les entretiens, par qui, et comment sont-ils restitués ?

Là aussi, la diversité prévaut : certains entretiens sont individuels, d'autres sont collectifs, mais ces différences sont effacées. Dans le cas d'entretiens collectifs, qui pose quelle question? On devine parfois des changements de locuteurs, mais sans certitude. Avec ses chapitres sans auteur explicite, le livre dans son ensemble opère une forme de reconstruction a posteriori d'un sociologue individuel fictif, effaçant les intervieweurs. Pour le lecteur, tout se passe comme si un seul et même intervieweur intervenait, alors que la variété des statuts, des expériences, des capitaux sociaux et des rapports à l'entrepreneuriat parmi les intervieweurs a pu avoir des effets sur

5. Lieu et conditions de l'entretien, extrait d'entretiens commentés, évocation du rapport enquêteur/enquêté.

6. Dubois F., Terral Ph. (2014), « Entrepreneurs passionnés », in Chauvin P.-M., Grossetti M., Zalio P.-P. (dir.), Dictionnaire sociologique de l'entrepreneuriat, Presses de Sciences Po, pp. 244-258. 
le déroulement et le contenu des interviews (ce que note d'ailleurs l'introduction générale). De fait, on voit apparaître au fil des entretiens des « styles » d'enquêteurs, notamment avec un degré de précision ou de naïveté variable, avec des questions maladroites ou répétitives, des relances inutiles, que les auteurs n'ont pas cherché à effacer. C'est probablement le corollaire négatif d'une grande qualité de cette recherche : sa dimension participative, impliquant chercheurs et apprentis chercheurs dans une entreprise commune non seulement d'enquête, mais aussi d'édition, ce qui est bien plus rare. 